University of Nebraska - Lincoln

DigitalCommons@University of Nebraska - Lincoln

$5-1-1998$

Invariant representations of finite rotation matrices and some applications

\author{
N. L. Manakov \\ Voronezh State University, manakov@phys.vsu.ru
}

A. V. Meremianin

Voronezh State University, meremianin@phys.vsu.ru

Anthony F. Starace

University of Nebraska-Lincoln, astarace1@unl.edu

Follow this and additional works at: https://digitalcommons.unl.edu/physicsstarace

Part of the Physics Commons

Manakov, N. L.; Meremianin, A. V.; and Starace, Anthony F., "Invariant representations of finite rotation matrices and some applications" (1998). Anthony F. Starace Publications. 63.

https://digitalcommons.unl.edu/physicsstarace/63

This Article is brought to you for free and open access by the Research Papers in Physics and Astronomy at DigitalCommons@University of Nebraska - Lincoln. It has been accepted for inclusion in Anthony F. Starace Publications by an authorized administrator of DigitalCommons@University of Nebraska - Lincoln. 


\title{
Invariant representations of finite rotation matrices and some applications
}

\author{
N. L. Manakov and A. V. Meremianin \\ Department of Physics, Voronezh State University, 394693 Voronezh, Russia \\ Anthony F. Starace \\ Department of Physics and Astronomy, The University of Nebraska, Lincoln, Nebraska 68588-0111
}

(Received 7 November 1997)

\begin{abstract}
Standard representations of finite rotation matrices (FRM) are defined by expressions that are wedded to two particular coordinate frames such as, e.g., are involved in the definition of Euler angles. We present here representations for the FRM in invariant tensor forms comprised of vectors defined in a space-fixed coordinate frame $K$. Three explicit expressions for a FRM are presented: First, in terms of tensor products of the spherical or Cartesian basis vectors of frame $K$, second in a differential form containing the tensor products of gradient operators, and, third, as the superposition of so-called "minimal" bipolar harmonics depending on any pair of unit vectors $\mathbf{a}, \mathbf{b}$ connected with a frame $K$. Based on these results for the FRM, the transformation rule for an irreducible tensor set under space rotations may be written in terms of bipolar harmonics. Our results are especially useful for analyzing angular distributions in atomic processes involving a precise accounting of all effects of photon and target polarizations. Four examples are considered as illustrations of the techniques presented. First, an invariant representation of the photon polarization tensor is found in terms of linear and circular polarization degrees of the photon beam. Second, an invariant decomposition of tripolar harmonics of second rank in terms of very simple, rank 2 tensors is presented. Third, a convenient parametrization is proposed for the polarization state multipoles of a polarized atomic target. Fourth, a simple invariant formula is derived for the angular distribution of polarized photons resulting from electric dipole photon emission by an arbitrary polarized atom. [S1050-2947(98)03305-8]
\end{abstract}

PACS number(s): 03.65.Ca, 03.65.Fd, 32.70.-n, 32.80.Fb

\section{INTRODUCTION}

The quantum theory of angular momentum is a powerful tool for investigations in atomic, molecular, and optical physics. Among the most important objects of this theory are the so-called finite rotation matrices $(\mathrm{FRM}), R_{m^{\prime} m}^{j}(\Omega)$, which describe the transformation of irreducible tensor sets under space rotations in accordance with the relation [1]

$$
T_{j m}^{\prime}=\sum_{m^{\prime}=-j}^{j} T_{j m^{\prime}} R_{m^{\prime} m}^{j}(\Omega),
$$

where $T_{j m}$ and $T_{j m}^{\prime}$ are the components of a tensor $T_{j}$, given in the "old" (space-fixed or initial) frame $K$ and in the "new" (rotated or laboratory or final) frame $K^{\prime}$, respectively. The symbol $\Omega$ denotes the rotation parameters. Equation (1) contains the FRM in an abstract form. Its explicit representation depends on the concrete choice of the parameters that specify the rotation.

There are two different representations of the FRM that are in wide use. These are the $D$ and the $U$ functions [1]. The Wigner functions $D_{m^{\prime} m}^{j}(\alpha \beta \gamma)$ depend on three Euler angles $\alpha, \beta, \gamma$, which describe the rotation in an elegant algebraic way. The functions $U_{m^{\prime} m}^{j}(\mathbf{n}, \omega)$ depend on the direction of the rotation axis $\mathbf{n}$ and on the rotation angle $\omega$. For this case the rotation parameters are $\Omega=\mathbf{n}, \omega$. Thus, one of the parameters is a vector with angular coordinates $\Theta, \Phi$, which are the same in both the frame $K$ and in the frame $K^{\prime}$. (Naturally, the angular coordinates of vectors that are not parallel to the n axis are different for frames $K$ and $K^{\prime}$.) Therefore, the explicit form of $U_{m^{\prime} m}^{j}$ depends upon the algebraic parameter $\omega$ and on $\Theta$ and $\Phi[1]$.

Despite the fact that the finite rotation matrix is the set of $(2 j+1)$ irreducible tensors numbered by either index $m^{\prime}$ or $m$ (i.e., in the frame $K^{\prime}$ they are tensors of rank $j$ with projections $m$ and in the frame $K$ they are tensors of rank $j$ with projections $m^{\prime}$ ), their (above-mentioned) explicit expressions do not have a structure that is obviously invariant, i.e., independent of a particular coordinate reference frame.

Even in the simplest case when the rank $j$ of a FRM is equal to unity, the functions $D_{m^{\prime} m}^{j}$ and $U_{m^{\prime} m}^{j}$ cannot be expressed as invariant combinations of some vectors. Only for $D_{0 m}^{j}$ with integer rank $j$ is such an invariant expression known. It is given by [1]

$$
D_{0 m}^{j}(0, \beta, \gamma)=\sqrt{\frac{4 \pi}{2 j+1}} Y_{j m}(\mathbf{a}),
$$

where $Y_{j m}(\mathbf{a})$ is the spherical harmonic of the unit vector a directed along the $Z$ axis of the space-fixed coordinate frame $K$; the two Euler angles, $\beta, \pi-\gamma$, are the polar angles of $\mathbf{a}$ in a frame $K^{\prime}$. As a consequence of Eq. (2), the Wigner $D$ function is also called a "generalized spherical harmonic.", Note that $U_{m^{\prime} m}^{j}(\mathbf{n}, \omega)$ has a representation as an expansion in spherical harmonics $Y_{l \mu}(\mathbf{n})$ with $0 \leqslant l \leqslant 2 j$, but its coefficients are the Clebsch-Gordan coefficients $C_{j m j m^{\prime}}^{l \mu}$, which depend upon $m, m^{\prime}[1,2]$. We use the term "invariant' for 
relations similar to Eq. (2) because the spherical harmonic on the right-hand side (rhs) of this identity is a tensor product of vectors a (cf. Sec. II).

Together with Racah techniques, the FRM's in the form of the Wigner functions are most useful for calculating atomic, molecular, and nuclear structures. But for studying angular distributions, especially for reactions involving polarized particles (in which case a large number of vectors enter the problem), the invariant representation of the FRM's as a combination of tensor products of vectors involved in the problem may often be preferable. Indeed, the angular distributions are invariant under coordinate rotations and their description in terms of invariant combinations of vectors characterizing the problem is very useful. Another important feature of the invariant representation of the FRM's is that it allows the representation of any tensor in an invariant form as a linear combination of FRM elements denumerated by an index $m^{\prime}$ [see Eq. (1)] with numerical coefficients that are the components of the same tensor in a suitable coordinate frame.

In this paper we present invariant forms for the finite rotation matrices that depend only on the vectors that are appropriate for a particular physical process being investigated. Specifically, in any physical process there exist one set of vector quantities that describes the initial state of the system and another set of vector quantities that describes the final state of the system. Various combinations of such vector quantities may be chosen to define in a natural way appropriate coordinate frames for the system. The description of a given physical process thus naturally involves a finite rotation matrix that describes the transformation from an initial coordinate frame to an appropriate final coordinate frame. Because the coordinate frames are defined by vectors that characterize the physical system, these vectors may be used to construct the finite rotation matrix rather than parameters specific to a particular coordinate reference frame. Indeed, the FRM constructed in this way may be evaluated in any particular pair of coordinate frames.

To derive the invariant form of the FRM we will describe the rotation in terms of angular coordinates of some vectors without any algebraic parameters similar to Euler angles or a rotation angle $\omega$, which are not inherent vector objects. Namely, we fix a vector set in an "old" or initial frame $K$, which may include the spherical or Cartesian basis vectors or, more generally, any pair of noncollinear vectors $\mathbf{a}, \mathbf{b}$ with fixed angle $\theta$ between them $(0<\theta<\pi$ is a free parameter). The angular coordinates of these vectors in a "new" or final frame $K^{\prime}$ are connected with those in $K$ by a rotation, whose explicit form need not be specified. Although a rotation is described by only three independent parameters (e.g., by the Euler angles) and for each concrete specification of vectors in frame $K$ the connection between the new and old coordinates of these vectors can be established and three independent parameters can be designated [see, e.g., Eq (69) below], such concretization of the rotation is unnecessary in most cases, and an invariant form for the FRM is quite sufficient. For this reason, we do not specify in our representations for the FRM the argument $\Omega$ in terms of some algebraic variables.
As we demonstrate below, in general the FRM can be presented as a superposition of irreducible tensors $\hat{\mathcal{M}}_{j m}^{s}(\Omega)$ of rank $j$ numbered by label $s$ and composed of some vectors fixed in an "old" frame $K$,

$$
R_{m^{\prime} m}^{j}(\Omega)=\sum_{s} c_{s}\left(j, m^{\prime}\right) \hat{\mathcal{M}}_{j m}^{s}(\Omega)
$$

Thus, the entire dependence of $R_{m^{\prime} m}^{j}$ on the tensor index $m$ is given by the tensor projections of $\hat{\mathcal{M}}_{j m}^{s}$, while the coefficients $c_{s}$ in Eq. (3) are independent of $m$. This is a most important fact, demonstrating an invariance of Eq. (3) with respect to a concrete choice of "new" coordinate frame $K^{\prime}$. Furthermore, Eq. (3) leads to an invariant parametrization (a "modified" transformation rule) for any irreducible tensor,

$$
T_{j m}^{\prime}=\sum_{s} t_{s}(j) \hat{\mathcal{M}}_{j m}^{s}(\Omega),
$$

where the coefficients $t_{s}(j)$, given by

$$
t_{s}(j)=\sum_{m^{\prime}} c_{s}\left(j, m^{\prime}\right) T_{j m^{\prime}},
$$

are linear combinations of the components $T_{j m^{\prime}}$ in an appropriate "old" frame $K$. Thus, the tensors $\hat{\mathcal{M}}_{j m}^{s}$ form an irreducible basis set in the space of irreducible tensors of rank $j$ similar to the spherical unit tensors of rank 1 .

In Sec. II we begin by considering the simplest case of FRM's with unit rank, $j=1$, and provide an explanation of our method for the construction of the FRM's in an invariant form. Specifically, an expansion of the FRM is derived in terms of spherical basis vectors in frame $K$. The sum on $s$ in Eq. (3) for this case contains only one operator $\hat{\mathcal{M}}_{j m}^{m^{\prime}}$ composed of spherical basis vectors [see Eq. (17)].

In Sec. III we derive a differential representation for FRM's as the result of the action of a tensor product of $\nabla_{\mathbf{r}}$ operators on spherical harmonics depending on angles of an auxiliary vector $\mathbf{r}$. The differential form of the FRM is very compact and it is useful for illustrating some general properties of the finite rotation matrix. In particular, the identities in Eqs. (24) and (25) demonstrate the high symmetry of $R_{m^{\prime} m}^{j}$ with respect to the indicies $m$ and $m^{\prime}$ in spite of the fact that these indices have a different meaning in our considerations: the first of them has a tensorial sense in the $K^{\prime}$ frame and the second one in the $K$ frame. Using the differential form of the FRM, in Sec. IV we find both a representation of $R_{m^{\prime} m}^{j}$ in terms of Cartesian basis vectors [see Eq. (36)] and two additional differential forms for the FRM containing $\nabla_{\mathbf{r}}$ operators and the scalar products of either Cartesian [see Eq. (33)] or spherical [see Eq. (39)] basis vectors with an auxiliary vector $\mathbf{r}$.

The expressions for FRM's presented in Secs. II and IV contain rather complicated combinations of basis vectors. Therefore in Sec. V we present the results in an alternative, compact form by representing the operators $\hat{\mathcal{M}}_{j m}^{s}$ in Eq. (3) as the special tensor products of two spherical harmonics (the so-called "minimal" bipolar harmonics $\mathcal{Y}_{j m}^{s}(\mathbf{a}, \mathbf{b})$ with $s=0, \ldots, j[3])$ depending on any pair of vectors $\mathbf{a}, \mathbf{b}$, con- 
nected with the frame $K$. We consider both the case in which a, b are the Cartesian basis vectors [see Eqs. (41) and (42)] and also the most general case in which $\mathbf{a}, \mathbf{b}$ are any pair of noncollinear vectors with an angle $\theta$ between them [see Eqs. (47) and (48)]. Note that in the last case the coefficients $c_{s}$ in Eq. (3) are $\theta$ dependent. For $m^{\prime}=0$ the superposition of "minimal", bipolar harmonics in Eq. (3) reduces immediately to Eq. (2). Thus, it seems that the term "generalized bipolar harmonics" for the FRM in invariant form is more appropriate than is the term "generalized spherical harmonics" for the Wigner $D$ functions.

In Sec. VI we illustrate the application of the invariant forms of the FRM's to a number of physical problems. As a particularly simple application, based on the differential form of the FRM, we first derive an invariant form [see Eq. (52)] for the photon polarization tensor of second rank in terms of the photon wave vector $\mathbf{k}$ and the unit vector $\hat{\boldsymbol{\epsilon}}$ directed along the major axis of the photon's polarization ellipse. Also very important for applications is a modified transformation rule [see Eq. (53)] for irreducible tensor sets, which follows from Eq. (1) after substitution of the expansion in Eq. (3) for $R_{m^{\prime} m}^{j}$ in terms of $\mathcal{Y}_{j m}^{s}$. Equation (53) confirms a general statement of Ref. [3] that the "minimal", harmonics $\mathcal{Y}_{j m}^{s}(\mathbf{a}, \mathbf{b})$ at fixed $j$ and variable $s$ form a convenient complete basis set for irreducible tensors having rank $j$.

Other applications we discuss are the following: In Sec. VI A we demonstrate a reduction technique for tripolar harmonics depending on three vectors $\mathbf{n}_{i}, i=1,2,3$. Such objects appear in kinematic analysis of reactions with 3 or more particles [e.g., $(e, 2 e)$ or $(\gamma, n e)$ processes]. As an example, we present in Eq. (57) an expression for tripolar harmonics of second rank in terms of tensors of rank 2 composed of 2 or 3 vectors $\mathbf{n}_{i}$. In Sec. VI B we derive a convenient, invariant parametrization for polarization state multipoles of a polarized target in terms of the Cartesian basis vectors $\mathbf{a}, \mathbf{b}, \mathbf{c}$ of a coordinate frame $K$ connected with a concrete process of preparing the polarized target. These results are used in Sec. VI C for the analysis of electric dipole photon emission by an arbitrary polarized atom with detection of photon polarization. We present the angular distribution of the polarized photons for this fundamental optical process in the simplest invariant form [see Eq. (68)], containing only the scalar products of basis vectors $\mathbf{a}, \mathbf{b}$, c, the wave vector $\mathbf{k}$, the photon polarization vectors $\mathbf{e}, \mathbf{e}^{*}$, and 8 initial parameters describing the target polarization. These four examples presented in Sec. VI demonstrate the efficiency of using invariant FRM's to analyze complex physical problems.

\section{INVARIANT REPRESENTATION OF THE FRM IN TERMS OF SPHERICAL BASIS VECTORS}

Consider first as an example the simplest case of a FRM $R_{m m^{\prime}}^{j}(\Omega)$ with $j=1$, which describes the transformation of vector operators under rotations. It is well known that an arbitrary vector $\mathbf{x}$ may be expanded in a spherical basis as follows:

$$
\mathbf{x}=\sum_{\mu=0, \pm 1}(-1)^{\mu} x_{-\mu} \mathbf{e}_{\mu}
$$

where $x_{\mu}=\left(\mathbf{x} \cdot \mathbf{e}_{\mu}\right)$ are the spherical components of $\mathbf{x}$ and as the spherical unit vectors we can use three arbitrary vectors $\mathbf{e}_{\mu} \quad$ satisfying the orthogonality condition $\left(\mathbf{e}_{\mu} \cdot \mathbf{e}_{\nu}\right)$ $=(-1)^{\mu} \delta_{\mu,-\nu}$, where $\mu, \nu=0, \pm 1$, and where $\delta_{\mu,-\nu}$ is the Kronecker delta. Equation (6) has an invariant form since it is valid in an arbitrary coordinate frame. Supposing $\mathbf{e}_{\mu}$ are the spherical basis vectors of the frame $K$ and multiplying Eq. (6) by the spherical basis vectors, $\mathbf{e}_{\nu}^{\prime}$, of the frame $K^{\prime}$, we have

$$
x_{\nu}^{\prime}=\sum_{\mu, \nu=0, \pm 1}(-1)^{\mu} x_{-\mu}\left(\mathbf{e}_{\mu}\right)_{\nu}
$$

where $x_{\nu}^{\prime}$ and $x_{\mu}$ are the components of $\mathbf{x}$ in the frames $K^{\prime}$ and $K$, respectively, and $\left(\mathbf{e}_{\mu}\right)_{\nu}$ are the components of the basis vectors $\mathbf{e}_{\mu}$ of $K$ with respect to the $K^{\prime}$ frame. Comparison of Eq. (7) with Eq. (1) yields

$$
R_{\mu \nu}^{1}(\Omega)=(-1)^{\mu}\left(\mathbf{e}_{-\mu}\right)_{\nu}=\left(\mathbf{e}_{\mu}^{*}\right)_{\nu} .
$$

Here the index $\mu$ enumerates the basis vectors $\mathbf{e}$, and $\nu$ is a tensor component index. This identity may be alternatively written as

$$
\begin{gathered}
R_{-1 \nu}^{1}(\Omega)-R_{1 \nu}^{1}(\Omega)=\sqrt{2}\left(\mathbf{e}_{x}\right)_{\nu}, \\
R_{-1 \nu}^{1}(\Omega)+R_{1 \nu}^{1}(\Omega)=i \sqrt{2}\left(\mathbf{e}_{y}\right)_{\nu}, \\
R_{0 \nu}^{1}(\Omega)=\left(\mathbf{e}_{z}\right)_{\nu},
\end{gathered}
$$

where $\mathbf{e}_{x, y, z}$ are Cartesian unit vectors of the frame $K$. Thus, the above expression for $R_{\mu \nu}^{1}(\Omega)$ has an invariant vector structure that is completely similar to the well-known Cartesian matrix, $a_{i k}$, of transformation between $K$ and $K^{\prime}$ in terms of direction cosines.

To derive an invariant expression for FRM with an arbitrary rank $j$ we note that any tensor composed of vectors fixed in the frame $K$ and equal to $R_{m^{\prime} m}^{j}(\Omega)$ in some coordinate frame $\bar{K}$ will coincide with $R_{m^{\prime} m}^{j}(\Omega)$ in any other frame. In particular, if some tensor $\bar{R}_{m^{\prime} m}^{j}(0)$ is equal to $R_{m^{\prime} m}^{j}(0)$ at $\bar{K}=K$, then the identity

$$
\bar{R}_{m^{\prime} m}^{j}(\Omega)=R_{m^{\prime} m}^{j}(\Omega)
$$

is valid also for an arbitrary orientation of the laboratory coordinate frame. This statement is based on rotation invariance arguments. Let a tensor $\bar{R}_{m^{\prime} m}^{j}(\Omega)$ coincide with $R_{m^{\prime} m}^{j}(0)$ at $\bar{K}=K$, then

$$
\sum_{m^{\prime}} T_{j m^{\prime}} R_{m^{\prime} m}^{j}(0)=\sum_{m^{\prime}} T_{j m^{\prime}} \bar{R}_{m^{\prime} m}^{j}(0) .
$$

Acting on both sides of this equation by the finite rotation operator $\hat{\mathcal{R}}(\Omega)$ and taking into account that $\hat{\mathcal{R}}(\Omega)$ does not act on the $T_{j m^{\prime}}$ since they are tensor components given in the space-fixed frame $K$, we obtain

$$
\sum_{m^{\prime}} T_{j m^{\prime}} \hat{\mathcal{R}}(\Omega) R_{m^{\prime} m}^{j}(0)=\sum_{m^{\prime}} T_{j m^{\prime}} \hat{\mathcal{R}}(\Omega) \bar{R}_{m^{\prime} m}^{j}(0),
$$


and, hence, $\bar{R}_{m^{\prime} m}^{j}(\Omega)=R_{m^{\prime} m}^{j}(\Omega)$. Thus, the problem is to find an invariant definition for the tensor equal to $R_{m^{\prime} m}^{j}(0)$ at $\bar{K}=K$.

Firstly we mention that in accordance with Eq. (1) the FRM for the case of zero rotation (i.e., when $K^{\prime}=K$ ) has the evident form

$$
R_{m^{\prime} m}^{j}(0)=\delta_{m^{\prime}, m}
$$

In order to define a tensor composed of basis vectors in a frame $K$ and satisfying Eq. (11) we introduce the special notation (cf. Chap. 3 of Ref. [1]) for a tensor product of $j$ identical vectors a

$$
\{\mathbf{a}\}_{j m}=\left\{\cdots\left\{\cdots\{\mathbf{a} \otimes \mathbf{a}\}_{2} \otimes \cdots \mathbf{a}\right\}_{j-1} \otimes \mathbf{a}\right\}_{j m} .
$$

The standard definitions of angular momentum theory [1] are used throughout the text. It is known [3] that the tensor $\{\mathbf{a}\}_{j m}$ is the same for an arbitrary coupling scheme on the righthand side of Eq. (12). Note that the spherical harmonic $Y_{j m}(\mathbf{r} / r)$ can be represented also as the tensor product of $j$ real vectors $\mathbf{r} / r[1]$ :

$$
Y_{j m}(\mathbf{r} / r)=\sqrt{\frac{(2 j+1) ! !}{4 \pi j !}} \frac{1}{r^{j}}\{\mathbf{r}\}_{j m} .
$$

In the coordinate frame with the $Z$ axis directed along vector $\mathbf{r}$ this expression reduces to

$$
Y_{j m}(0,0)=\delta_{m, 0} \sqrt{\frac{2 j+1}{4 \pi}} .
$$

Now we note that in the frame $K$ the tensor product in Eq. (12) composed of spherical basis vectors has the form

$$
\left\{\mathbf{e}_{ \pm 1}\right\}_{k q}=(-1)^{k} \delta_{k, \mp q},
$$

where $\mathbf{e}_{ \pm 1}=\mp(1 / \sqrt{2})(\mathbf{b} \pm i \mathbf{c})$ are the spherical basis vectors; $\mathbf{a}, \mathbf{b}, \mathbf{c}$ are the Cartesian basis vectors directed along the axes $Z, X, Y$ of the frame $K$, respectively. The identity (15) can be derived by straightforward calculations taking into account that $C_{a a b b}^{a+b a+b}=1$ and

$$
\left(\mathbf{e}_{0}\right)_{\mu}=\delta_{\mu, 0}, \quad\left(\mathbf{e}_{\nu}\right)_{\mu}=-\delta_{\mu,-\nu}, \quad \nu= \pm 1, \quad \mu=0, \pm 1
$$

for spherical components of vectors $\mathbf{e}_{ \pm 1}$ and $\mathbf{e}_{0} \equiv \mathbf{a}$.

By direct calculations we can verify that the tensor $R_{k m}^{j}(\Omega)$ defined by the equation

$$
R_{ \pm k m}^{j}(\Omega)=A_{j k}\left\{\left\{\mathbf{e}_{\mp 1}\right\}_{k} \otimes\left\{\mathbf{e}_{0}\right\}_{j-k}\right\}_{j m}, \quad k \geqslant 0
$$

coincides with the FRM after an appropriate choice of a normalization factor $A_{j k}$. Indeed, it can be clearly seen from Eqs. (12) and (15) that in the $K$ frame $R_{ \pm k m}^{j}(\Omega=0)$ has the form

$$
R_{ \pm k m}^{j}(0)=(-1)^{k} C_{k k j-k 0}^{j k} \sqrt{\frac{(j-k) !}{(2 j-2 k-1) ! !}} A_{j k} \delta_{m, \pm k} .
$$

Hence, $R_{ \pm k m}^{j}(\Omega)$ satisfies the condition (11) and therefore coincides with the FRM in accordance with the above arguments. Substituting the explicit form for the Clebsh-Gordan coefficient $C_{k k j-k 0}^{j k}$ in Eq. (18), we find for $A_{j k}$ the following expression:

$$
A_{j k}=(-1)^{k} \sqrt{\frac{2^{k-j}(2 j) !}{(j+k) !(j-k) !}} .
$$

Equation (17) is the simplest form of an invariant representation for the FRM, because the coefficients $A_{j k}$ do not depend on the tensor projection index $m$, unlike the case of the $U(\mathbf{n}, \omega)$-function representation of the FRM [2]. Note that the index $k$ in Eq. (17) is not a tensor index with respect to the laboratory frame $K^{\prime}$. In this frame $k$ just enumerates the basis tensors $R_{ \pm k m}^{j}$, in a way similar to that of the index $\nu$ in the case of the spherical basis vectors $\mathbf{e}_{\nu}$ for the $K$ frame.

\section{INVARIANT DIFFERENTIAL FORM OF FRM}

Below we present an invariant form of the FRM that results from the action of a special differential operator on the standard spherical harmonic. Our treatment is based on the use of invariant rank-decreasing operators for spherical harmonics [3]. For these operators the following identity is valid:

$$
\left\{\hat{O}_{k}^{l-}(r, \nabla) \otimes Y_{l}(\mathbf{r} / r)\right\}_{q m}=\delta_{q, l-k} Y_{l-k, m}(\mathbf{r} / r), \quad k \leqslant l .
$$

For the explicit form of $\hat{O}_{k \mu}^{l-}(r, \nabla)$ see Eq. (A6) in Ref. [3]. As we describe below, the use of this identity at $l=k \equiv j$ $(q=0)$ leads to an important formal relation:

$$
T_{j m}^{\prime}=(-1)^{j} \sqrt{4 \pi(2 j+1)} \hat{O}_{j m}^{j-}(r, \nabla)\left[T_{j}^{\prime} \cdot Y_{j}(\mathbf{r} / r)\right],
$$

where $T_{j}^{\prime}$ is an arbitrary tensor of integer rank $j$ and $\mathbf{r}$ is an arbitrary vector, and we consider this equation in a rotated frame $K^{\prime}$. While it appears that $T_{j m}^{\prime}$ on the left-hand side of Eq. (21) depends on $\mathbf{r}$ through the spherical harmonic $Y_{j k}(\mathbf{r} / r)$, this dependence is removed by the gradient operators, which constitute $\hat{O}_{j m}^{l-}(r, \nabla)$ with $j=l$ as follows [3]:

$$
\hat{O}_{j m}^{j-}(r, \nabla)=\frac{(-1)^{j}}{\sqrt{(2 j+1) j !(2 j+1) ! !}}\{\nabla\}_{j m} r^{j} .
$$

The identity in Eq. (21) may be verified by direct calculation as follows: 


$$
\begin{aligned}
\hat{O}_{j m}^{j-}(r, \nabla)\left[T_{j}^{\prime} \cdot Y_{j}(\mathbf{r} / r)\right] & =\sum_{k=-j}^{j}(-1)^{k} \hat{O}_{j m}^{j-}(r, \nabla) T_{j-k}^{\prime} Y_{j k}(\mathbf{r} / r) \\
& =\sum_{k, s, m_{s}}(-1)^{k} T_{j-k}^{\prime} C_{j m j k}^{s m_{s}}\left\{\hat{O}_{j}^{j-}(r, \nabla) \otimes Y_{j}(\mathbf{r} / r)\right\}_{s m_{s}}=(-1)^{j} \frac{1}{\sqrt{4 \pi(2 j+1)}} T_{j m}^{\prime} .
\end{aligned}
$$

Here the first equality follows immediately from the definition of the scalar product for two tensors, $T_{j}^{\prime}$ and $Y_{j}$, the second one from the orthogonality properties of ClebshGordan coefficients, and the third one from Eq. (20).

Since a scalar product of tensors does not depend on the orientation of a coordinate frame, we can consider the scalar product on the right-hand side of Eq. (21) as defined in the space-fixed frame $K$ (instead of the laboratory frame $K^{\prime}$ ), and, hence, the replacement $T_{j}^{\prime} \rightarrow T_{j}$ should be made on the right-hand side of Eq. (21). Of course, the spherical harmonic $Y_{j}(\mathbf{r} / r)$ must also be replaced by its definition in the frame $K$. We thus arrive at the following identity:

$$
T_{j m}^{\prime}=\sum_{k=-j}^{j} T_{j k}(-1)^{j+k} \sqrt{4 \pi(2 j+1)} \hat{O}_{j m}^{j-}(r, \nabla) Y_{j-k}(\mathbf{r} / r) .
$$

Comparing this result with Eq. (1) and using Eq. (22), we derive the "differential" representation of $R_{k m}^{j}(\Omega)$ :

$$
R_{k m}^{j}(\Omega)=\sqrt{\frac{4 \pi}{j !(2 j+1) ! !}}\{\nabla\}_{j m} r^{j} Y_{j k}^{*}(\mathbf{r} / r) .
$$

As may be seen from this identity, the FRM $R_{k m}^{j}(\Omega)$ is a tensor in the frame $K^{\prime}$ with respect to the index $m$, and a complex-conjugated tensor in the frame $K$ with respect to the index $k$. Moreover, taking into account the auxiliary relation [4],

$$
\{\nabla\}_{j m} r^{j} Y_{j k}^{*}(\mathbf{r} / r)=\{\nabla\}_{j k}^{*} r^{j} Y_{j m}(\mathbf{r} / r),
$$

we obtain another expression, similar to Eq. (24):

$$
R_{k m}^{j}(\Omega)=\sqrt{\frac{4 \pi}{j !(2 j+1) ! !}}\{\nabla\}_{j k}^{*} r^{j} Y_{j m}(\mathbf{r} / r) .
$$

Here the spherical harmonic is defined in the laboratory frame $K^{\prime}$, and the tensor product of gradient operators is defined in the space-fixed frame $K$. From Eqs. (24) and (25) it follows that, although indices $k$ and $m$ have a different meaning, there is an explicit symmetry between them.

These equations are convenient also for explicit demonstration of fundamental properties of the FRM's, such as unitarity, group properties, etc. As an example, we derive here an expression for $R_{m^{\prime} m}^{j}(\Omega)$, where $\Omega=\Omega^{\prime} \cdot \Omega^{\prime \prime}$ is the product of the rotations $K \rightarrow K^{\prime} \rightarrow K^{\prime \prime}$ described by the parameters $\Omega^{\prime}, \Omega^{\prime \prime}$. Replacement of the spherical function $Y_{j m^{\prime}}^{*}(\mathbf{r} / r)$ in Eq. (24), which is defined in the frame $K$, by the expression $\Sigma_{m^{\prime \prime}} R_{m^{\prime} m^{\prime \prime}}^{j}\left(\Omega^{\prime}\right) Y_{j m^{\prime \prime}}^{*}(\mathbf{r} / r)$, where $Y_{j m^{\prime \prime}}$ is defined in the frame $K^{\prime}$, leads to the relation:

$$
\begin{aligned}
R_{m^{\prime} m}^{j}(\Omega)= & (-1)^{j} \sqrt{4 \pi(2 j+1)} \\
& \times \sum_{m^{\prime \prime}} R_{m^{\prime} m^{\prime \prime}}^{j}\left(\Omega^{\prime}\right) \hat{O}_{j m}^{j-}(r, \nabla) Y_{j m^{\prime \prime}}^{*}(\mathbf{r} / r) .
\end{aligned}
$$

Taking into account Eq. (24), we verify explicitly the wellknown group identity

$$
R_{m^{\prime} m}^{j}(\Omega)=\sum_{m^{\prime \prime}} R_{m^{\prime} m^{\prime \prime}}^{j}\left(\Omega^{\prime}\right) R_{m^{\prime \prime} m}^{j}\left(\Omega^{\prime \prime}\right)
$$

\section{REPRESENTATION OF THE FRM IN TERMS OF CARTESIAN BASIS VECTORS}

Below we demonstrate that the result of the action of gradient operators on the spherical harmonic $Y_{j k}^{*}(\mathbf{r} / r)$ in Eq. (24) can be calculated in terms of tensor constructions depending on the unit vectors $\mathbf{a}, \mathbf{b}$ directed along the $Z, X$ axes of the $K$ frame, respectively. Thus we shall find the explicit form of the FRM in terms of the Cartesian basis vectors of the space-fixed frame $K$. Our considerations below depend upon two known facts concerning the spherical harmonics.

(i) It is well known that the spherical harmonic $Y_{j-k}(\mathbf{r} / r)=(-1)^{k} Y_{j k}^{*}(\mathbf{r} / r)$ can be written in the standard form [1]

$$
\begin{aligned}
Y_{j-k}(\mathbf{r} / r)= & \sqrt{\frac{2 j+1}{4 \pi} \frac{(j-k) !}{(j+k) !}}[\sin \theta \exp (-i \phi)]^{k} \\
& \times P_{j}^{(k)}(\cos \theta), \quad k>0,
\end{aligned}
$$

where $\theta$ is an angle between the vectors $\mathbf{r}$ and $\mathbf{a}, \cos \theta$ $=(\mathbf{r} \cdot \mathbf{a}) / r$, and $\phi$ is an angle between the $X$ axis and the projection of the vector $\mathbf{r}$ on the $X-Y$ plane of the system $K$,

$$
\cos \phi=\frac{(\mathbf{r} \cdot \mathbf{b})}{r \sin \theta}, \quad \sin \phi=\frac{(\mathbf{r} \cdot \mathbf{c})}{r \sin \theta} .
$$

The polynomial $P_{j}^{(k)}(x)=d^{k} P_{j}(x) / d x^{k}$ is the $k$ th derivative of the Legendre polynomial $P_{j}(x)$.

(ii) The following useful identity,

$$
\cos k \phi=\frac{k}{2} \sum_{s=0}^{[k / 2]}(-1)^{s} \frac{(k-s-1) !}{s !(k-2 s) !}(2 \cos \phi)^{k-2 s},
$$

follows immediately from the fact that $\cos k \phi=T_{k}(\cos \phi)$ is the Tchebyshev polynomial of the first kind. An identity similar to Eq. (27) for $\sin k \phi$ can be derived by differentiation of Eq. (27) with respect to $\phi$.

Taking Eqs. (26) and (27) into account, one can rewrite ${ }^{j} Y_{j k}(\mathbf{r} / r)$ in terms of scalar products as 


$$
\begin{aligned}
r^{j} Y_{j-k}(\mathbf{r} / r)= & \sqrt{\frac{2 j+1}{4 \pi} \frac{(j-k) !}{(j+k) !}} r^{j-k} P_{j}^{(k)}(\mathbf{a} \cdot \mathbf{r} / r) \sum_{s=0}^{[k / 2]}(-1)^{s} \frac{(k-s-1) !}{s !(k-2 s) !}[2(\mathbf{b} \cdot \mathbf{r})]^{k-2 s-1}\left[r^{2}-(\mathbf{a} \cdot \mathbf{r})^{2}\right]^{s} \\
& \times[k(\mathbf{b} \cdot \mathbf{r})-i(k-2 s)(\mathbf{c} \cdot \mathbf{r})], \quad k>0 .
\end{aligned}
$$

Note that the gradient operators in Eq. (24) do not act on the terms in Eq. (28) that which contain scalar products of $\mathbf{r}$ with itself owing to the following relation:

$$
\{\nabla\}_{l m}(\mathbf{r} \cdot \mathbf{r})^{k}=0, \quad k<l .
$$

Thus, one should keep in Eq. (28) only the terms from the expansion of $r^{j-k} P_{j}^{(k)}(\mathbf{a} \cdot \mathbf{r} / r)$ into a power series in $(\mathbf{a} \cdot \mathbf{r})$ that do not contain any powers of $(\mathbf{r} \cdot \mathbf{r})$. This includes in particular the term $\left[(\mathbf{r} \cdot \mathbf{r})-(\mathbf{a} \cdot \mathbf{r})^{2}\right]^{s}$. Thus the following substitutions should be made in Eq. (28):

$$
\left[r^{2}-(\mathbf{a} \cdot \mathbf{r})^{2}\right]^{s} \rightarrow(-1)^{s}(\mathbf{a} \cdot \mathbf{r})^{2 s},
$$

and

$$
r^{j-k} P_{j}^{(k)}(\mathbf{a} \cdot \mathbf{r} / r) \rightarrow \frac{(2 j-1) ! !}{(j-k) !}(\mathbf{a} \cdot \mathbf{r})^{j-k}
$$

As explained below, it is more convenient to consider two symmetrized combinations of $R_{k m}^{j}(\Omega)$ [cf. Eq. (8)]:

$$
\begin{gathered}
R_{k m}^{j+}(\Omega)=R_{-k m}^{j}(\Omega)+(-1)^{k} R_{k m}^{j}(\Omega) \\
R_{k m}^{j-}(\Omega)=-i\left[R_{-k m}^{j}(\Omega)-(-1)^{k} R_{k m}^{j}(\Omega)\right], \quad k>0 .
\end{gathered}
$$

Substitution of Eqs. (28), (30), and (31) into Eq. (24) yields for $R_{k m}^{j+}(\Omega)$,

$$
R_{k m}^{j+}(\Omega)=\sqrt{\frac{(2 j-1) ! !}{j !(j-k) !(j+k) !}} \sum_{s=0}^{[k / 2]} \frac{k(k-s-1) !}{s !(k-2 s) !}\{\nabla\}_{j m}(\mathbf{r} \cdot \mathbf{a})^{j-k+2 s}[2(\mathbf{r} \cdot \mathbf{b})]^{k-2 s} .
$$

The right-hand side of this equation may be calculated explicitly, using the identity

$$
\{\nabla\}_{j m}(\mathbf{r} \cdot \mathbf{a})^{j-k+2 s}(\mathbf{r} \cdot \mathbf{b})^{k-2 s}=j !\left\{\{\mathbf{a}\}_{j-k+2 s} \otimes\{\mathbf{b}\}_{k-2 s}\right\}_{j m},
$$

which follows immediately from the Leibniz rule for differentiation of products and from the simple relations

$$
\begin{gathered}
\nabla(\mathbf{r} \cdot \mathbf{a})^{n}=n \mathbf{a}(\mathbf{r} \cdot \mathbf{a})^{n-1}, \quad n>0, \\
\{\nabla \otimes \mathbf{r}\}_{2 m}=0 .
\end{gathered}
$$

Thus, $R_{k m}^{j+}(\Omega)$ can be presented in the following final form:

$$
R_{k m}^{j+}=\sqrt{\frac{j !(2 j-1) ! !}{(j-k) !(j+k) !}} \sum_{s=0}^{[k / 2]} 2^{k-2 s} \frac{k(k-s-1) !}{s !(k-2 s) !}\left\{\left\{\mathbf{a}_{j-k+2 s} \otimes\{\mathbf{b}\}_{k-2 s}\right\}_{j m} .\right.
$$

An analogous identity for $R_{k m}^{j-}(\Omega)$ can be derived similarly:

$$
R_{k m}^{j-}=\sqrt{\frac{j !(2 j-1) ! !}{(j-k) !(j+k) !}} \sum_{s=0}^{[(k-1) / 2]} 2^{k-2 s} \frac{(k-s-1) !}{s !(k-2 s-1) !}\left\{\mathbf{c} \otimes\left\{\{\mathbf{a}\}_{j-k+2 s} \otimes\{\mathbf{b}\}_{k-2 s-1}\right\}_{j-1}\right\}_{j m} .
$$

For completeness, we obtain Eq. (17) of Sec. II using the differentiation method. Firstly, note that the term $\sin \theta \exp (-i \phi)$ on the rhs of Eq. (26) can be rewritten as

$$
\sin \theta(\cos \theta-i \sin \theta)=\sqrt{2} \frac{\left(\mathbf{e}_{-1} \cdot \mathbf{r}\right)}{r},
$$

where $\mathbf{e}_{-1}$ is the spherical unit vector in frame $K$. Secondly, replacing $P_{j}^{(k)}(\cos \theta)$ in Eq. (26) in accordance with Eq. (31), we obtain one more differential representation of the FRM in terms of spherical basis vectors

$$
R_{k m}^{j}(\Omega)=(-1)^{k} \sqrt{\frac{2^{k}(2 j-1) ! !}{j !(j+k) !(j-k) !}}\{\nabla\}_{j m}\left(\mathbf{e}_{0} \cdot \mathbf{r}\right)^{j-k}\left(\mathbf{e}_{-} \cdot \mathbf{r}\right)^{k}, \quad k>0 .
$$


This equation demonstrates clearly the independence of the final results on an auxiliary vector $\mathbf{r}$ owing to the fact that $\mathbf{r}$ appears to the $j$ th power. Calculating the action of the gradient operators in this equation, using Eqs. (34) and (35), we arrive immediately at Eq. (17) of Sec. II.

\section{EXPANSION OF A FRM INTO A BASIS OF BIPOLAR HARMONICS}

Some preliminary remarks concerning the bipolar harmonics $(\mathrm{BH})$ are in order. These objects are defined by the following equation [1]:

$$
Y_{j m}^{l l^{\prime}}\left(\mathbf{n}, \mathbf{n}^{\prime}\right)=\left\{\mathrm{Y}_{l}(\mathbf{n}) \otimes \mathrm{Y}_{l^{\prime}}\left(\mathbf{n}^{\prime}\right)\right\}_{j m} .
$$

As was shown in [3], for the case when $l+l^{\prime}>j$, the $\mathrm{BH}$ may be expanded into a superposition of "minimal" BH's $\mathcal{Y}_{j m}^{k}\left(\mathbf{n}, \mathbf{n}^{\prime}\right)$, which are defined by

$$
\mathcal{Y}_{j m}^{k}\left(\mathbf{n}, \mathbf{n}^{\prime}\right)=\left\{\mathrm{Y}_{j-k}(\mathbf{n}) \otimes \mathrm{Y}_{k}\left(\mathbf{n}^{\prime}\right)\right\}_{j m},
$$

where $k=0, \ldots, j$. The harmonics $\mathcal{Y}_{j m}^{k}$ have some important properties. Namely, the Clebsh-Gordan coefficient entering the tensor product in Eq. (40) can be written in explicit form as a product of factorials without any sums. Further, as was demonstrated in [3], the minimal BH's form a "linearly independent'" set in the space of tensors with fixed rank $j$, i.e., there are no linear relations of the kind

$$
\sum_{k} C_{j k}(\cos \theta) \mathcal{Y}_{j m}^{k}\left(\mathbf{n}, \mathbf{n}^{\prime}\right)=0
$$

where the $C_{j k}(\cos \theta)$ are scalar coefficients depending on the scalar product $\left(\mathbf{n} \cdot \mathbf{n}^{\prime}\right)=\cos \theta$. This means that the minimal BH's $\mathcal{Y}_{j m}^{k}\left(\mathbf{n}, \mathbf{n}^{\prime}\right)$ are the simplest irreducible tensors of fixed rank $j$, which can be constructed of two vectors $\mathbf{n}, \mathbf{n}^{\prime}$. The number of linearly independent minimal BH's of rank $j$ is evidently equal to $(j+1)$. It is important to note that the minimal BH's are polar tensors for each $k$, since under space inversion (i.e., when $\mathbf{n}, \mathbf{n}^{\prime} \rightarrow-\mathbf{n},-\mathbf{n}^{\prime}$ ) they acquire the additional phase factor $(-1)^{j}$, which is independent of $k$.

Now an expansion of the FRM using a basis of minimal BH's can be obtained by substitution of Eq. (13) for the spherical harmonics into Eqs. (36) and (37):

$$
\begin{gathered}
R_{k m}^{j+}(\Omega)=\sum_{s=0}^{[k / 2]} A_{k s}^{(0)} \mathcal{Y}_{j m}^{k-2 s}(\mathbf{a}, \mathbf{b}), \\
R_{k m}^{j-}(\Omega)=\sum_{s=0}^{[(k-1) / 2]} A_{k s}^{(1)}\left\{\mathbf{c} \otimes \mathcal{Y}_{j-1}^{k-2 s-1}(\mathbf{a}, \mathbf{b})\right\}_{j m},
\end{gathered}
$$

where the coefficients $A_{k s}^{(\lambda)}$ have the form

$$
A_{k s}^{(\lambda)}=4 \pi \frac{[k(1-\lambda)+\lambda](k-s-1) !}{2^{3 s} s !} \sqrt{\frac{2^{3 k-\lambda} j !(2 j-1) ! !(j-k+2 s) !}{(j-k) !(j+k) !(2 k-4 s-2 \lambda+1) !(2 j-2 k+4 s+1) ! !}}, \quad k>0
$$

Equations (41) and (42) are expansions of the FRM in a basis of minimal bipolar harmonics depending on the orthogonal vectors a,b,c. It is important to note that the number $2 j+1$ of different BH's entering the expansion of the FRM is the same as the total number of functions $R_{m^{\prime} m}^{j}(\Omega)$ with different indices $m^{\prime}$ at fixed $m$. Since an arbitrary tensor $T_{j m}^{\prime}$ in a laboratory frame is a superposition of elements of FRM's [see Eq. (1)], it is clear that $T_{j m}^{\prime}$ can be expressed also as a combination of minimal BH's [see Eq. (53) below]. Note that the functions $R_{k m}^{j+}(\Omega), R_{k m}^{j-}(\Omega)$, defined by Eqs. (41) and (42) are polar tensors. Also, there is an essential difference between the two combinations $R_{k m}^{j+}(\Omega)$ and $R_{k m}^{j-}(\Omega)$. Namely, $R_{k m}^{j+}(\Omega)$ is invariant with respect to inversion of the $Y$ axis of the space-fixed frame $K$ while $R_{k m}^{j-}(\Omega)$ changes sign.

Generally, it is possible to derive an expansion of the FRM's in BH's depending on any pair of vectors fixed in the frame $K$. This result follows from the simple relation for a tensor product composed of two arbitrary vectors

$$
\left\{c_{1} \mathbf{a}_{1}+c_{2} \mathbf{a}_{2}\right\}_{j m}=\sum_{n=0}^{j}\left(\begin{array}{l}
j \\
n
\end{array}\right) c_{1}^{j-n} c_{2}^{n}\left\{\left\{\mathbf{a}_{1}\right\}_{j-n} \otimes\left\{\mathbf{a}_{2}\right\}_{n}\right\}_{j m},
$$

where $\left(\begin{array}{l}n \\ j\end{array}\right)$ is the binomial coefficient and $\mathbf{a}_{1}, \mathbf{a}_{2}$ are two arbitrary vectors. Equation (44) can be verified using Eq. (12) and the fact that a tensor product is a linear function of each tensor entering the product. Taking into account Eq. (13), Eq. (44) can be rewritten as

$$
\begin{aligned}
\mid c_{1} \mathbf{a}_{1} & +\left.c_{2} \mathbf{a}_{2}\right|^{j} Y_{j m}(\mathbf{v}) \\
& =\sum_{n=0}^{j} \sqrt{\frac{4 \pi(2 j+1) !}{(2 n+1) !(2 j-2 n+1) !}} c_{1}^{j-n} c_{2}^{n} \mathcal{Y}_{j m}^{n}\left(\mathbf{a}_{1}, \mathbf{a}_{2}\right),
\end{aligned}
$$

where $\mathbf{v}$ is the unit vector directed along the vector $c_{1} \mathbf{a}_{1}$ $+c_{2} \mathbf{a}_{2}$. Thus, we have derived, by a most simple method, the so-called addition theorem for solid harmonics $[1,5]$, which are the products $r^{j} Y_{j m}(\mathbf{r} / r)$.

Below we present one of the most convenient invariant representations of the FRM's as a superposition of BH's depending on a pair of nonorthogonal unit vectors $\mathbf{n}, \mathbf{n}^{\prime}$. Let the vector $\mathbf{n}$ be directed along the $Z$ axis of the space-fixed frame $K$, and the vector $\mathbf{n}^{\prime}$ lie in the same plane as the vector $\mathbf{b}$. In other words, 


$$
\mathbf{a}=\mathbf{n}, \quad \mathbf{b}=\frac{\mathbf{n}^{\prime}-\mathbf{n} \cos \theta}{\sin \theta}, \quad \mathbf{c}=\frac{\left[\mathbf{n} \times \mathbf{n}^{\prime}\right]}{\sin \theta},
$$

where $\theta$ is the angle between vectors $\mathbf{n}$ and $\mathbf{n}^{\prime}$. Of course, the angle $\theta$ must be nonzero, otherwise the basis Eq. (46) fails.

Substituting Eq. (44) into Eqs. (41) and (42), noting that in our case $c_{1}=1 / \sin \theta$ and $c_{2}=-\cot \theta$, and performing a summation over one free index, we arrive at the following identities:

$$
R_{k m}^{j+}(\Omega)=\sum_{s=0}^{k} B_{k s}^{(0)}(\theta) \mathcal{Y}_{j m}^{s}\left(\mathbf{n}, \mathbf{n}^{\prime}\right)
$$

and

$$
R_{k m}^{j-}(\Omega)=\sum_{s=0}^{k-1} B_{k s}^{(1)}(\theta)\left\{\left[\mathbf{n} \times \mathbf{n}^{\prime}\right] \otimes \mathcal{Y}_{j-1}^{s}\left(\mathbf{n}, \mathbf{n}^{\prime}\right)\right\}_{j m},
$$

where the coefficients $B_{k s}^{(\lambda)}(\theta)$ are given by

$$
B_{k s}^{(\lambda)}(\theta)=\frac{4 \pi}{(\sin \theta)^{k}} C_{k-s-\lambda}^{1 / 2-k}(\cos \theta) \frac{2[k(1-\lambda)+\lambda](k+s+\lambda-1) !}{(2 k-1) ! !} \sqrt{\frac{2^{s}(j-s-\lambda) ! j !(2 j-1) ! !}{(2 s+1) !(2 j-2 s-2 \lambda+1) ! !(j+k) !(j-k) !}}
$$

It is important to note that the Gegenbauer polynomials $C_{k-s-\lambda}^{1 / 2-k}(\theta)$ on the rhs of Eq. (49) do not depend on the rank $j$ of the FRM's. We note also that half the coefficients $B$ at $\theta=\pi / 2$ coincide with the coefficients $A$ [cf. Eq. (43)]:

$$
B_{k k-2 s-\lambda}^{(\lambda)}(\pi / 2)=A_{k s}^{(\lambda)},
$$

and the others are zero:

$$
B_{k k-2 s-\lambda+1}^{(\lambda)}(\pi / 2)=0 .
$$

Thus, the representations in Eqs. (41) and (42) are seen to be special cases of the more general expansions in Eqs. (47) and (48).

We note that Eqs. (47) and (48) show the function $R_{k m}^{j+}(\Omega)$ to be a polar tensor and the function $R_{k m}^{j-}(\Omega)$ to be an axial tensor [because the tensor product in Eq. (48) contains an axial vector $\left.\left[\mathbf{n} \times \mathbf{n}^{\prime}\right]\right]$. This latter result contrasts with Eq. (42) for $R_{k m}^{j-}(\Omega)$. However, there is no contradiction with the tensor transformation rule in Eq. (1). Namely, the tensor components of one and the same tensor $T_{j}$ on the rhs of Eq. (1) may be either scalars or pseudoscalars depending on whether the unit vector c of the space-fixed frame $K$ is either a polar or an axial vector.

We note finally that if we define the "generalized", spherical harmonic $\widetilde{Y}_{j m}(\mathbf{e})$ of complex unit vector e by the tensor product in Eq. (13) with $\mathbf{r} / r \rightarrow \mathbf{e}$, then Eq. (17) can be rewritten [using Eqs. (19) and (40)] as

$$
\begin{aligned}
R_{ \pm k m}^{j}(\Omega) & =c(k, j)\left\{\widetilde{Y}_{k}\left(\mathbf{e}_{\mp 1}\right) \otimes Y_{j-k}\left(\mathbf{e}_{0}\right)\right\}_{j m} \\
& =c(k, j) \widetilde{\mathcal{Y}}_{j m}^{j-k}\left(\mathbf{e}_{\mp 1}, \mathbf{e}_{0}\right),
\end{aligned}
$$

where

$$
c(k, j)=(-1)^{k} 4 \pi \sqrt{\frac{(2 j) ! k ! 2^{k-j}}{(j+k) !(2 k+1) ! !(2 j-2 k+1) ! !}} .
$$

When $k=0$ this equation reduces immediately to Eq. (2). Thus, the FRM's may be considered as the "generalized" minimal BH's of the spherical basis vectors of the old frame $K$.

\section{SOME APPLICATIONS}

The different invariant representations of the FRM's we have presented [cf. Eqs. (17), (24), (25), (33), (36), (37), (39), (47), and (48)] each have their uses in particular applications. Thus, although Eqs. (47) and (48) are very general, in some situations the use of the differential representations in Eqs. (24) and (25) of the FRM may prove simpler. For example, if an explicit expression for a scalar product of a tensor $T_{j m}$ and a spherical harmonic is known in terms of scalar products of vectors, then Eqs. (23) and (24) allow one to obtain an explicit form of $T_{j m}$ in terms of tensor products of vectors. Before describing the general procedure for using invariant FRM's in concrete applications, we give an example of the use of the simpler Eqs. (23) and (24).

Consider the photon polarization tensor $T_{p m}$ $=\left\{\mathbf{e} \otimes \mathbf{e}^{*}\right\}_{p m}$, where e is the unit (complex) photon polarization vector (see, e.g., [7]). Invariant explicit forms of $T_{p m}$ are known only for $p=0,1$ and are given by

$$
T_{0}=-\frac{1}{\sqrt{3}}, \quad T_{1}=\frac{1}{\sqrt{2}} \xi \hat{\mathbf{k}},
$$

where $\xi$ is the degree circular polarization of the photon,

$$
\xi=i \hat{\mathbf{k}} \cdot\left[\mathbf{e} \times \mathbf{e}^{*}\right]
$$

and $\hat{\mathbf{k}}$ is the unit vector along the direction of the photon beam. The following explicit expressions for $T_{2 m}$ are used widely in applications:

$$
T_{20}=-\frac{1}{\sqrt{6}}, \quad T_{2 \pm 1}=0, \quad T_{2 \pm 2}=\frac{1}{\sqrt{2}}\left(\xi_{3} \pm \xi_{1}\right),
$$


where $\xi_{1,3}$ are the standard Stokes parameters [8]. These identities are valid, of course, only in a coordinate frame where the $Z$ axis is directed along the vector $\hat{\mathbf{k}}$.

To derive an invariant expression for $T_{2 m}$ we note that, in accordance with Eqs. (13), (22), and (23), tensor $T_{2 m}$ may be written in the following form:

$$
T_{2 m}=\frac{1}{2}\{\nabla\}_{2 m}\left(\{\mathbf{r} \otimes \mathbf{r}\}_{2} \cdot\left\{\mathbf{e} \otimes \mathbf{e}^{*}\right\}_{2}\right)=\frac{1}{2}\{\nabla\}_{2 m}|\mathbf{e} \cdot \mathbf{r}|^{2} .
$$

Here the term $|\mathbf{e} \cdot \mathbf{r}|^{2}$ may be calculated in explicit form using the auxiliary relation [3]

$$
\begin{aligned}
2 \operatorname{Re}(\mathbf{e} \cdot \mathbf{r})\left(\mathbf{e}^{*} \cdot \mathbf{r}^{\prime}\right)= & 2 l(\mathbf{r} \cdot \hat{\boldsymbol{\epsilon}})\left(\hat{\boldsymbol{\epsilon}} \cdot \mathbf{r}^{\prime}\right) \\
& +(l-1)\left([\mathbf{r} \times \hat{\mathbf{k}}] \cdot\left[\hat{\mathbf{k}} \times \mathbf{r}^{\prime}\right]\right),
\end{aligned}
$$

where $\hat{\boldsymbol{\epsilon}}$ is the unit vector along the major axis of the photon polarization ellipse, and $l$ is the degree of linear polarization, $l=\mathbf{e} \cdot \mathbf{e}=\mathbf{e}^{*} \cdot \mathbf{e}^{*}$. Using this identity and calculating the action of the gradient operators in Eq. (50) we arrive at an invariant expression for $T_{2 m}$ :

$$
T_{2 m}=l\left\{\hat{\boldsymbol{\epsilon}} \otimes \hat{\boldsymbol{\epsilon}}_{\}_{2 m}}+\frac{l-1}{2}\{\hat{\mathbf{k}} \otimes \hat{\mathbf{k}}\}_{2 m},\right.
$$

which is valid in any coordinate frame. Equation (52) is valid also for a partially polarized photon beam with the degree of partial polarization $\mathcal{P}=1-l^{2}-\xi^{2}$. So the results presented are equivalent to the photon density matrix approach, but they have an explicit invariant form and therefore can be more convenient for analyzing photon polarization effects in the angular distributions.

The regular method for application of the invariant representations for the FRM's to concrete problems is as follows: Insert Eqs. (47) and (48) into Eq. (1) to obtain a "modified" transformation rule for the irreducible tensorial sets:

$$
\begin{aligned}
T_{j m}^{\prime}= & \sum_{s=0}^{j} T_{j, s}^{(0)} \mathcal{Y}_{j m}^{s}\left(\mathbf{n}, \mathbf{n}^{\prime}\right)+\sum_{s=0}^{j-1} T_{j, s}^{(1)} \\
& \times\left\{\left[\mathbf{n} \times \mathbf{n}^{\prime}\right] \otimes \mathcal{Y}_{j-1}^{s}\left(\mathbf{n}, \mathbf{n}^{\prime}\right)\right\}_{j m},
\end{aligned}
$$

where the scalar coefficients $T_{j, s}^{(\lambda)}, \lambda=0,1$ are defined by

$$
\begin{gathered}
T_{j, s}^{(0)}=\sum_{k=s}^{j} B_{k, s}^{(0)}(\theta)\left[T_{j-k}+(-1)^{k} T_{j k}\right] / 2, \\
T_{j, s}^{(1)}=i \sum_{k=s+1}^{j} B_{k, s}^{(1)}(\theta)\left[T_{j-k}-(-1)^{k} T_{j k}\right] / 2,
\end{gathered}
$$

where $B_{00}^{(0)}(\theta)=4 \pi /(\sqrt{2 j+1})$, the other $B_{m, n}^{(\lambda)}$ coefficients are defined by the general Eq. (49), and the $T_{j k}$ are the components of the tensor $T_{j}$ in an appropriate coordinate frame $K$ most suitable for a concrete problem. All applications of invariant representations of FRM's are based on use of Eq. (53), as illustrated in some examples below.

\section{A. Simplification of tripolar and multipolar harmonics with small external ranks}

We use here Eqs. (53) and (54) to calculate tripolar harmonics, which appear, e.g., in the analysis of angular distributions for $(e, 2 e)$ processes with emission or absorption of a photon, for Compton scattering by bound electrons, etc. The tripolar harmonic is the tensor product of three spherical harmonics [1].

$$
Y_{j m}^{l_{1},\left(l_{2} l_{3}\right) l}\left(\mathbf{n}_{1}, \mathbf{n}_{2}, \mathbf{n}_{3}\right)=\left\{Y_{l_{1}}\left(\mathbf{n}_{1}\right) \otimes\left\{Y_{l_{2}}\left(\mathbf{n}_{2}\right) \otimes Y_{l_{3}}\left(\mathbf{n}_{3}\right)\right\}_{l}\right\}_{j m} .
$$

In the coordinate frame with the $Z$ axis along $\mathbf{n}_{1}$ and the $Y$ axis along $\left[\mathbf{n}_{1} \times \mathbf{n}_{2}\right]$ we have

$$
\begin{aligned}
& Y_{j m}^{l_{1},\left(l_{2} l_{3}\right) l}\left(00, \theta_{2} 0, \theta_{3} \phi_{3}\right) \\
& =\sqrt{\frac{2 l_{1}+1}{4 \pi}} C_{l_{1} 0 l_{m}}^{j m} \sum_{m_{2} m_{3}} C_{l_{2} m_{2} l_{3} m_{3}}^{l m} Y_{l_{2} m_{2}}\left(\theta_{2} 0\right) \\
& \quad \times Y_{l_{3} m_{3}}\left(\theta_{3} \phi_{3}\right),
\end{aligned}
$$

where $\theta_{2,3}$ are the angles between the vector $\mathbf{n}_{1}$ and the vectors $\mathbf{n}_{2,3}$, respectively, and $\phi_{3}$ is the angle between two planes defined by vectors $\mathbf{n}_{1}, \mathbf{n}_{2}$ and $\mathbf{n}_{1}, \mathbf{n}_{3}$.

The use of Eqs. (53) and (54) leads to the representation of the tripolar harmonic in Eq. (55) in an arbitrary coordinate frame in terms of the simpler objects in Eq. (56) and tensors of rank $j$ composed from a minimal number of vectors $\mathbf{n}_{1}$, $\mathbf{n}_{2}, \mathbf{n}_{3}$. For simplicity, we present below the results for the most important case in applications, tripolar harmonics of rank 2:

$$
\begin{aligned}
Y_{2 m}^{l_{1},\left(l_{2} l_{3}\right) l}\left(\mathbf{n}_{1}, \mathbf{n}_{2}, \mathbf{n}_{3}\right)= & a_{1}\left\{\mathbf{n}_{1} \otimes \mathbf{n}_{1}\right\}_{2 m}+a_{2}\left\{\mathbf{n}_{2} \otimes \mathbf{n}_{2}\right\}_{2 m} \\
& +a_{3}\left\{\mathbf{n}_{1} \otimes \mathbf{n}_{2}\right\}_{2 m}+b_{1}\left\{\left[\mathbf{n}_{1} \times \mathbf{n}_{2}\right]\right. \\
& \left.\otimes \mathbf{n}_{1}\right\}_{2 m}+b_{2}\left\{\left[\mathbf{n}_{1} \times \mathbf{n}_{2}\right] \otimes \mathbf{n}_{2}\right\}_{2 m}
\end{aligned}
$$

where the coefficients $a_{i}, b_{i}$ are given by

$$
\begin{aligned}
& a_{1}=\sqrt{\frac{3}{2}} Y_{20}+\frac{\cos \theta_{1}}{\sin \theta_{1}}\left[Y_{21}+(-1)^{\lambda} p Y_{21}^{*}\right] \\
& +\frac{\cos ^{2} \theta_{1}+1}{2 \sin ^{2} \theta_{1}}\left[Y_{22}+(-1)^{\lambda_{p}} Y_{22}^{*}\right], \\
& a_{2}=\frac{1}{\sin ^{2} \theta_{1}}\left[Y_{22}+(-1)^{\lambda_{p}} Y_{22}^{*}\right], \\
& \begin{aligned}
a_{3}= & -\frac{1}{\sin \theta_{1}}\left[Y_{21}+(-1)^{\lambda_{p}} Y_{21}^{*}\right] \\
& -\frac{2 \cos \theta_{1}}{\sin ^{2} \theta_{1}}\left[Y_{22}+(-1)^{\lambda} p Y_{22}^{*}\right],
\end{aligned}
\end{aligned}
$$




$$
\begin{aligned}
b_{1}= & i\left(\frac{1}{\sin \theta_{1}}\left[Y_{21}-(-1)^{\lambda} p Y_{21}^{*}\right]\right. \\
& \left.+\frac{\cos \theta_{1}}{\sin ^{2} \theta_{1}}\left[Y_{22}-(-1)^{\lambda_{p}} Y_{22}^{*}\right]\right), \\
b_{2}= & -i \frac{1}{\sin ^{2} \theta_{1}}\left[Y_{22}-(-1)^{\lambda} p Y_{22}^{*}\right] .
\end{aligned}
$$

For conciseness we have introduced in Eq. (58) the abbreviation $Y_{2 m}=Y_{2 m}^{l_{1},\left(l_{2} l_{3}\right) l}\left(00, \theta_{2} 0, \theta_{3} \phi_{3}\right)$. The index $\lambda_{p}=l_{1}$ $+l_{2}+l_{3}$ denotes the parity of the tripolar harmonic, i.e., for even $\lambda_{p}$ the harmonic is a polar tensor and for odd $\lambda_{p}$ it is an axial tensor (pseudotensor). As follows from Eq. (58), for even $\lambda_{p}$ the coefficients $a_{i}$ are scalars and the $b_{i}$ are pseudoscalars, and for odd $\lambda_{p}$ the scalar characters are reversed. The coefficients $a, b$ satisfy an important symmetry relation:

$$
a_{1}, b_{1} \rightleftharpoons a_{2}, b_{2} \quad \text { for } \quad \mathbf{n}_{2} \rightleftharpoons \mathbf{n}_{3} .
$$

This relation implies the following changes in the ranks and arguments of the spherical harmonics in Eq. (58): $l_{2}, \theta_{2} \rightleftharpoons l_{3}, \theta_{3}, 0 \rightarrow \phi_{2}$, and $\phi_{3} \rightarrow 0$, where $\phi_{2}$ is the angle between the planes $\mathbf{n}_{1}, \mathbf{n}_{3}$ and $\mathbf{n}_{1}, \mathbf{n}_{2}$.

Finally, we note that the results in Eqs. (57) and (58) can be generalized easily for the case of an arbitrary $N$-polar harmonic of rank 2 depending on $N$ vectors $\mathbf{n}_{i}$. For this purpose the parameter $\lambda_{p}$ should be changed to $\lambda_{p}=\Sigma_{n} l_{n}$, where the $l_{n}$ are the ranks of the spherical harmonics which enter the multipolar harmonic, and instead of $Y_{2 k}$ one should substitute the components of the multipolar harmonic in an appropriate fixed coordinate frame. For the case $N>3$ any three vectors from the set $\mathbf{n}_{i}$ with $i=1, \ldots, N$ can be used as the vectors $\mathbf{n}_{1,2,3}$ in Eq. (57); the coefficients $a_{i}$ and $b_{j}$ in Eq. (57) will then depend on the relative angles of all vectors in the problem. Thus the tensor structure given in Eq. (57) is independent of the number $N$.

\section{B. Invariant form of polarization momenta}

Equations (41) and (42) for the FRM and the transformation rule in Eq. (53) may be useful even for those cases in which an invariant expression for some tensor is unknown. As an example we consider here the irreducible components, $P_{r m}$, of the density matrix for some mixed state of a quantum system $[6,7]$, whose matrix elements are defined by the equation

$$
\left\langle J M|\rho| J M^{\prime}\right\rangle=\sum_{r m}(-1)^{J-M} C_{J M^{\prime} J-M}^{r m} P_{r m},
$$

where $\rho$ is a density matrix operator. We suppose, for simplicity, that the states with the momenta $J^{\prime} \neq J$ do not contribute in the statistical mixture. For this case we have

$$
P_{r m}^{*}=(-1)^{m} P_{r-m},
$$

and the following important relation is valid:

$$
P_{r m}=2^{r} \sqrt{\frac{(2 r+1) ! !(2 J-r) !}{r !(2 J+r+1) !}}\left\langle\{\mathbf{J}\}_{r m}\right\rangle,
$$

where $\left\langle\{\mathbf{J}\}_{r m}\right\rangle$ denotes an averaged value of the tensor product of angular momentum operators $\mathbf{J}$ [7]. The convenience of the tensors $P_{r m}$ consists in the fact that one is able in many cases to take into account an explicit symmetry of the problem (e.g., for spherically symmetric states only $P_{00}$ is nonzero, etc.). The tensors $P_{r m}$, also called "polarization momenta" [8], are connected with the standard polarization state multipoles $\rho_{r m}$, namely, $P_{r m}=\rho_{r m}^{*}$.

Since the polarization multipoles are tensors, the transformation rule in Eq. (53) is applicable. Below we present explicit expressions for polarization multipoles with ranks $r=0,1,2$. The use of Eqs. (41) and (42) in Eq. (53) for $P_{r m}$ yields

$$
\begin{gathered}
P_{0}=\frac{1}{\sqrt{2 J+1}} \\
\mathbf{P}_{1}=\mathcal{P}_{10} \mathbf{a}+\mathcal{P}_{11} \mathbf{b}+\mathcal{P}_{11}^{\prime} \mathbf{c} \\
P_{2}=\mathcal{P}_{20}\{\mathbf{a} \otimes \mathbf{a}\}_{2}+\mathcal{P}_{22}\{\mathbf{b} \otimes \mathbf{b}\}_{2}+\mathcal{P}_{21}\{\mathbf{a} \otimes \mathbf{b}\}_{2}+\mathcal{P}_{21}^{\prime}\{\mathbf{a} \otimes \mathbf{c}\}_{2} \\
+\mathcal{P}_{22}^{\prime}\{\mathbf{b} \otimes \mathbf{c}\}_{2},
\end{gathered}
$$

where a,b,c are the Cartesian unit vectors along the $Z, X, Y$ axes of the frame $K$, which may be connected, evidently, with symmetry properties of an excitation process used for the preparation of the target polarization. The parameters $\mathcal{P}_{r m}$ are also combinations of polarization tensor components $\bar{P}_{r m}$ in the frame $K$. For brevity, we omit the component index $m$ in the tensor notation $P_{r m}$. The $(2 r+1)$ different angular combinations in Eqs. (62) and (63) are equal in number to the number of independent parameters $\mathcal{P}_{r m}$. These parameters have the form

$$
\begin{gathered}
\mathcal{P}_{10}=\bar{P}_{10}, \quad \mathcal{P}_{11}=-\sqrt{2} \operatorname{Re} \bar{P}_{11}, \quad \mathcal{P}_{11}^{\prime}=-\sqrt{2} \operatorname{Im} \bar{P}_{11}, \\
\mathcal{P}_{20}=\sqrt{\frac{3}{2}} \bar{P}_{20}+\operatorname{Re} \bar{P}_{22}, \quad \mathcal{P}_{21}=-2 \operatorname{Re} \bar{P}_{21}, \\
\mathcal{P}_{22}=2 \operatorname{Re} \bar{P}_{22}, \\
\mathcal{P}_{21}^{\prime}=-2 \operatorname{Im} \bar{P}_{21}, \quad \mathcal{P}_{22}^{\prime}=2 \operatorname{Im} \bar{P}_{22} .
\end{gathered}
$$

In accordance with Eq. (60), Eq. (64) may be rewritten as

$$
\begin{gathered}
\mathcal{P}_{10}=c_{1}\left\langle\mathbf{J}_{\mathbf{a}}\right\rangle, \quad \mathcal{P}_{11}=c_{1}\left\langle\mathbf{J}_{\mathbf{b}}\right\rangle, \quad \mathcal{P}_{11}^{\prime}=c_{1}\left\langle\mathbf{J}_{\mathbf{c}}\right\rangle, \\
\mathcal{P}_{20}=c_{2}\left(\left\langle\mathbf{J}_{\mathbf{a}}^{2}\right\rangle-\left\langle\mathbf{J}_{\mathbf{c}}^{2}\right\rangle\right), \quad \mathcal{P}_{22}=c_{2}\left(\left\langle\mathbf{J}_{\mathbf{b}}^{2}\right\rangle-\left\langle\mathbf{J}_{\mathbf{c}}^{2}\right\rangle\right), \\
\mathcal{P}_{21}=c_{2}\left\langle\mathbf{J}_{\mathbf{a}} \mathbf{J}_{\mathbf{b}}\right\rangle, \quad \mathcal{P}_{21}^{\prime}=c_{2}\left\langle\mathbf{J}_{\mathbf{a}} \mathbf{J}_{\mathbf{c}}\right\rangle, \quad \mathcal{P}_{22}^{\prime}=c_{2}\left\langle\mathbf{J}_{\mathbf{b}} \mathbf{J}_{\mathbf{c}}\right\rangle,
\end{gathered}
$$

where the constants $c_{1,2}$ are

$$
c_{1}=\sqrt{\frac{3}{J(J+1)(2 J+1)}},
$$

$$
c_{2}=\sqrt{\frac{30}{J(J+1)(2 J-1)(2 J+1)(2 J+3)}} .
$$


It is seen from these identities that the parameters $\mathcal{P}_{10}, \mathcal{P}_{11}$, $\mathcal{P}_{11}^{\prime}$ are proportional to the projections of the "mean angular momentum" $\langle\mathbf{J}\rangle$ on the Cartesian basis vectors of the abovediscussed coordinate frame $K$. There furthermore exists an explicit connection between the parameters $\mathcal{P}_{r m}$ and the symmetry properties of a polarized state. For example, if a polarized state has an axial symmetry, then, directing the vector a (or b) along the symmetry axis, we obtain the result that only tensors containing coefficients $\mathcal{P}_{r 0}\left(\right.$ or $\left.\mathcal{P}_{r r}\right)$ contribute to $P_{r m}$. For the case of right-left symmetric polarized states (i.e., with respect to the exchange $\mathbf{c} \rightleftharpoons-\mathbf{c}$ ), all the tensors with coefficients $\mathcal{P}^{\prime}$ vanish.

\section{Emission of photons by polarized atoms}

As an application of Eqs. (61)-(63) to solving concrete problems we consider the emission of photons by polarized atoms. Using the electric-dipole approximation in length gauge, an angular distribution $d w_{\hat{\mathbf{k}} \mathbf{e}} / d \Omega$ of the photons emitted by a polarized atom in the direction $\hat{\mathbf{k}}$ can be written as [8]

$$
\frac{d w_{\hat{\mathbf{k}} \mathbf{e}}}{d \Omega}=\frac{\omega^{3}}{2 \pi \hbar c^{3}} T_{f i},
$$

where

$$
\begin{aligned}
T_{f i}= & \sum_{M_{i} M_{i}^{\prime} M_{f}}\left\langle E_{f} J_{f} M_{f}|(\mathbf{e} \cdot \mathbf{d})| E_{i} J_{i} M_{i}\right\rangle \\
& \times\left\langle E_{f} J_{f} M_{f}|(\mathbf{e} \cdot \mathbf{d})| E_{i} J_{i} M_{i}^{\prime}\right\rangle *\left\langle J_{i} M_{i}\left|\rho^{i}\right| J_{i} M_{i}^{\prime}\right\rangle .
\end{aligned}
$$

Here $\mathbf{e}$ and $\mathbf{d}$ are the photon polarization vector and dipole momentum operator, respectively, and $\rho^{i}$ is the density matrix operator. The use of standard angular momentum algebra leads to the following expression for $T_{f i}$ :

$$
\begin{aligned}
& T_{f i}=(-1)^{J_{i}+J_{f}}\left|\left\langle E_{f} J_{f}\|\mathbf{d}\| E_{i} J_{i}\right\rangle\right|^{2} \\
& \times \sum_{p=0,1,2}\left\{\begin{array}{ccc}
1 & 1 & p \\
J_{i} & J_{i} & J_{f}
\end{array}\right\}\left(P_{p} \cdot\left\{\mathbf{e} \otimes \mathbf{e}^{*}\right\}_{p}\right),
\end{aligned}
$$

where the tensors $P_{p m}$ are defined by Eq. (59). The scalar products in the rhs of this equation may be easily calculated according to Eqs. (61)-(64). Finally, we obtain

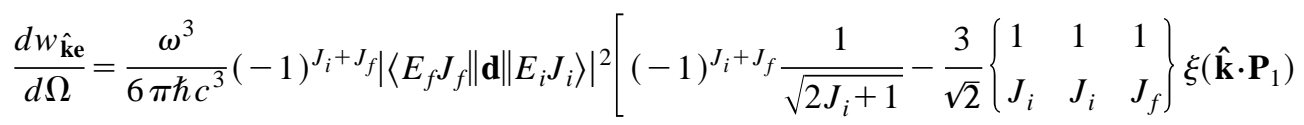

$$
\begin{aligned}
& +\left\{\begin{array}{ccc}
1 & 1 & 2 \\
J_{i} & J_{i} & J_{f}
\end{array}\right\}\left[\mathcal{P}_{20}\left(3|\mathbf{e} \cdot \mathbf{a}|^{2}-1\right)+\mathcal{P}_{22}\left(3|\mathbf{e} \cdot \mathbf{b}|^{2}-1\right)+3 \mathcal{P}_{21} \operatorname{Re}(\mathbf{e} \cdot \mathbf{a})\left(\mathbf{e}^{*} \cdot \mathbf{b}\right)+3 \mathcal{P}_{21}^{\prime} \operatorname{Re}(\mathbf{e} \cdot \mathbf{a})\left(\mathbf{e}^{*} \cdot \mathbf{c}\right)\right. \\
& \left.\left.+3 \mathcal{P}_{22}^{\prime} \operatorname{Re}(\mathbf{e} \cdot \mathbf{b})\left(\mathbf{e}^{*} \cdot \mathbf{c}\right)\right]\right] \text {. }
\end{aligned}
$$

This expression has a high symmetry and an invariant structure because it contains only the scalar products of vectors. The 8 parameters $\mathcal{P}$ describe the polarization properties of an initial atomic state with an arbitrary total angular momentum $J$. The identity (51) is sufficient for detailed analysis of photon polarization effects in terms of linear and circular polarization degrees. Note that the vector of "atomic orientation", $\mathbf{P}_{1}$ in Eq. (68) describes circular dichroism effects in the angular distribution, i.e., the difference between the intensities of emitted radiation with opposite helicities detected in the same direction $\hat{\mathbf{k}}$.

All particular experimental situations connected with atomic polarization also can be easily analyzed using Eqs. (68) and (65). For example, if an atomic ensemble has been excited due to the absorption of polarized photons, then it is convenient to choose the directions of the vectors $\mathbf{a}$ and $\mathbf{b}$ along respectively the main axis of the polarization ellipse for the incident photons and along the direction of the incident photon beam. For linearly polarized incident photons, the terms in Eq. (68) containing the vectors b, c must be omitted, unlike the case of pure circularly polarized photons, when the terms with a, c vanish. We do not analyze here other general features of the angular distribution $d w_{\hat{\mathbf{k}} \mathbf{e}} / d \Omega$ since they are evident from Eq. (68) and have been discussed previously [7,9].

\section{CONCLUDING REMARKS}

The invariant representations for the FRM [Eqs. (47) and (48)] together with the new transformation rule for the irreducible tensorial sets [Eq. (53)] are the key results of the present work. Our other results and the applications we have presented have been summarized in Sec. I. Here we discuss the problem of the choice of the rotation parameters corresponding to our invariant expressions for the FRM. Unlike the case of the usual $D$ or $U$ functions, for which the rotation is described by three real parameters (e.g., the Euler angles or the polar angles of vector $\mathbf{n}$ and the rotation angle $\omega$ ), we describe the rotation by the directions of two unit vectors $\mathbf{n}, \mathbf{n}^{\prime}$. The bipolar harmonics in Eqs. (47) and (48) are dependent on the spherical angles of the vectors $\mathbf{n}, \mathbf{n}^{\prime}$ given in the laboratory frame $K^{\prime}$. In the space-fixed frame $K$ these vectors have the form $\mathbf{n}=(0,0), \mathbf{n}^{\prime}=(\theta, 0)$, where $\theta$ is the angle 
between $\mathbf{n}, \mathbf{n}^{\prime}$. [We use the notation $\mathbf{a}=\left(\theta_{\mathbf{a}}, \phi_{\mathbf{a}}\right)$ for the angular coordinates of a unit vector $\mathbf{a}$, where $\theta_{\mathbf{a}}$ is the angle between $\mathbf{a}$ and the $Z$ axis, and where $\phi_{\mathrm{a}}$ is the angle between the projection of a on the plane $X-Y$ and the $X$ axis of the coordinate frame.] The polar angles of vectors $\mathbf{n}, \mathbf{n}^{\prime}$ in the frame $K^{\prime}$ can be connected with the standard Euler rotation angles $\alpha, \beta, \gamma$ as follows:

$$
\begin{gathered}
\theta_{\mathbf{n}}=\beta, \\
\phi_{\mathbf{n}}=\pi-\gamma,
\end{gathered}
$$

$\cos \theta_{\mathbf{n}^{\prime}}=\cos \theta \cos \beta+\sin \theta \sin \beta \cos \alpha$,

$$
\cot \left(\phi_{\mathbf{n}^{\prime}}+\gamma\right)=-\cot \alpha \cos \beta+\frac{\cot \theta \sin \beta}{\sin \alpha} .
$$

As we see from these equalities, only three parameters out of five (four spherical angles + one "free parameter" $\theta$ ) are independent.

The use of invariant representations of the FRM can be efficient not only in problems involving multipole expansions, where they enable one to extract the polarization and spin dependence of the cross sections in an invariant vector form, but also in cases when standard techniques of angular momentum and tensor algebra (e.g., the Wigner-Eckart theorem) are not applicable. For example, such a situation appears in the recent Ref. [10], in which the orientation effects in an electron-impact ionization of polarized atoms has been investigated using the so-called $3 C$ wave function, which has an asymptotic form that coincides with that of the exact three-body Coulomb wave function. In this work tensors $\Sigma_{K Q}$ describing the dynamics of the process have been calculated numerically in a special coordinate frame. The results in this paper allow one to write $\Sigma_{K Q}$ immediately in the following form:

$$
\Sigma_{K Q}=\sum_{N=0}^{K} C_{K N} \mathcal{Y}_{K Q}^{N}\left(\mathbf{n}_{1}, \mathbf{n}_{2}\right),
$$

which is valid even for the case of a nondiagonal density matrix for an initial atomic state. In Eq. (70) the $C_{K N}$ are scalar coefficients, dependent on both the dynamics of the process and on the angles between the vectors of the problem. The vectors $\mathbf{n}_{1,2}$ can be chosen as the unit noncollinear vectors along the directions of electron momenta.

An analogous situation appears also in Ref. [11], where the angular distributions in triple-electron photoionization have been investigated using a $6 C$ wave function, which is a generalization of the $3 C$ wave function used for double photoionization. In this case the results of Sec. V allow one to write the cross section in an invariant form, similar to Eq. (68).

\section{ACKNOWLEDGMENTS}

N.L.M. gratefully acknowledges the hospitality of the University of Nebraska-Lincoln where a part of this work was carried out during his visit to the Department of Physics and Astronomy. This research was supported in part by the Russian Fund for Basic Research and by NSF under Grant No. PHY-9722110.
[1] D. A. Varshalovich, A. N. Moskalev, and V. K. Khersonskii, Quantum Theory of Angular Momentum (World Scientific, Singapore, 1988).

[2] H. E. Moses, Ann. Phys. (N.Y.) 37, 224 (1966); M. S. Marinov, Sov. J. Nucl. Phys. 5, 943 (1967).

[3] N. L. Manakov, S. I. Marmo, and A. V. Meremianin, J. Phys. B 29, 2711 (1996).

[4] E. W. Hobson, The Theory of Spherical and Ellipsoidal Harmonics (Cambridge University Press, Cambridge, 1931).

[5] Sima Chakrabarti and D. P. Dewangan, J. Phys. B 28, L769 (1995).
[6] U. Fano, Rev. Mod. Phys. 29, 76 (1957).

[7] K. Blum, Density Matrix Theory and Applications (Plenum Press, New York, 1981).

[8] V. B. Berestetskii, E. M. Lifshitz, and L. P. Pitaevskii, Quantum Electrodynamics, 2nd ed. (Pergamon Press, Oxford, 1982).

[9] U. Fano and J. H. Macek, Rev. Mod. Phys. 45, 553 (1973).

[10] J. Berakdar, A. Engelns, and H. Klar, J. Phys. B 29, 1109 (1996).

[11] A. W. Malcherek and J. S. Briggs, J. Phys. B 30, 4419 (1997). 